\title{
A first model of stable magnetic configuration in stellar radiation zones
}

\author{
Vincent Duez ${ }^{1}$, Jonathan Braithwaite ${ }^{1}$ and Stéphane Mathis ${ }^{2}$ \\ ${ }^{1}$ Argelander Institut für Astronomie, Universität Bonn, Auf dem Hügel 71, D-53111 Bonn, \\ Germany, email: vduez@astro.uni-bonn.de, jonathan@astro.uni-bonn.de \\ ${ }^{2}$ Laboratoire AIM, CEA/DSM-CNRS-Université Paris Diderot, IRFU/SAp Centre de Saclay, \\ F-91191 Gif-sur-Yvette, France, email: stephane.mathis@cea.fr
}

\begin{abstract}
We test the stability of a magnetic equilibrium configuration using numerical simulations and semi-analytical tools. The tested configuration is, as described by Duez \& Mathis (2010), the lowest energy state for a given helicity in a stellar radiation zone. We show using 3D magneto-hydrodynamic (MHD) simulations that the present configuration is stable with respect to all submitted perturbations, that would lead to the development of kink-type instabilities in the case of purely poloidal or toroidal fields, both well known to be unstable. We also discuss, using semi-analytic work, the stabilizing influence of one component on the other and show that the found configuration actually lies in the stability domain predicted by a linear analysis of resonant modes.
\end{abstract}

Keywords. stars: magnetic fields, MHD

\section{Introduction}

The large-scale, ordered nature of magnetic fields detected at the surface of some Ap, $\mathrm{O}$ and $\mathrm{B}$ type stars and the scaling of their strengths (according to the flux conservation scenario) favour a fossil hypothesis, whose origin is not yet elucidated. To have survived since the stellar formation, a field must be stable on a dynamic (Alfvén) timescale. It was suggested by Prendergast (1956) that a stellar magnetic field in stable axisymmetric equilibrium must contain both poloidal (meridional) and toroidal (azimuthal) components, since both are unstable on their own (Tayler 1973; Wright 1973). This was confirmed recently by numerical simulations (Braithwaite \& Spruit 2004; Braithwaite \& Nordlund 2006) showing that an arbitrary initial field evolves on an Alfvén timescale into a stable configuration; axisymmetric mixed poloidal-toroidal fields were found. On the other hand, magnetic equilibria models displaying similar properties have been re-examined analytically by Duez \& Mathis (2010). We here address the question of their stability usng both numerical and semi-analytical tools as recently reported in Duez et al. (2010).

\section{The model}

We deal with non force-free magnetic configurations (i.e. with a non-zero Lorentz force) in equilibrium inside a conductive fluid in absence of convection. Several reasons inclined us to focus on non force-free equilibria. First, Reisenegger (2009) reminds us that no configuration can be force-free everywhere. Although there do exist "force-free" configurations, they must be confined by some region or boundary layer with non-zero or singular Lorentz force. Second, non force-free equilibria have been identified in plasma physics as the result of relaxation (self-organization process involving magnetic reconnections in resistive MHD), e.g. by Montgomery \& Phillips (1988). Third, as shown by Duez \& Mathis (2010), this family of equilibria is a generalization of Taylor states (force-free 
relaxed equilibria; see Taylor 1974) in a stellar context, where the stratification of the medium plays a crucial role. Finally, we know (Chandrasekhar 1958) that in the ideal MHD limit the mass encompassed in magnetic flux surfaces is conserved in the axisymmetric case. We hence assume here that it is roughly conserved during the non-ideal relaxation phase, which leads automatically to non-force-free states (Woltjer 1959).

The equilibrium obtained is described in detail in Duez \& Mathis (2010) as the lowest energy configuration conserving the invariants of the problem (during the relaxation phase) which are the magnetic helicity (preventing the rapid energy decay) and the mass enclosed in magnetic poloidal flux surfaces (to account for the non force-free property) which is due to the stable stratification.

\section{Stability numerical analysis}

We use the Stagger code (Nordlund \& Galsgaard 1995). We model the star as a selfgravitating ball of ideal gas $(\gamma=5 / 3)$ with radial density and pressure profiles initially obeying the polytropic relation $P \propto \rho^{1+(1 / n)}$, with index $\mathrm{n}=3$, therefore stably stratified. More details on the numerical model setup can be found in Braithwaite \& Nordlund 2006. The configuration is then submitted to white perturbations ( $1 \%$ in density). The dynamical evolution of the mixed configuration is compared to its purely poloidal and its purely toroidal components, whose behaviour are well known to be unstable due to kink-type instabilities. The magnetic and velocity amplitudes are plotted on Fig. 1. As

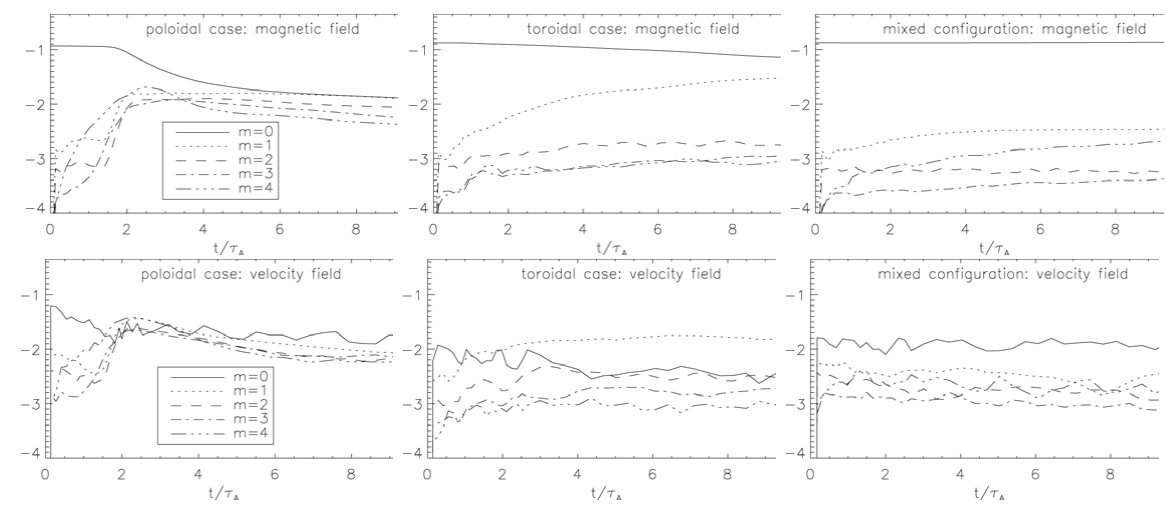

Figure 1. Time evolution of the (log) amplitudes in azimuthal modes $m=0$ to 4 averaged over the stellar volume of the magnetic field (top row) and the velocity field (bottom row) in the simulations with the purely poloidal field (left), purely toroidal field (middle) and the mixed field (right). Initially, all the magnetic energy is in the $m=0$ mode.

we can see, in contrast to these unstable configurations, the mixed poloidal-toroidal one does not exhibit any sign of instability, even for high azimuthal wavenumbers (up to about 40). The magnetic and velocity amplitudes are plotted on the right of fig. 1, where we see an absence of growing modes. The kinetic energy present results simply from the initial perturbation and the oscillations and waves it sets up.

To better examine the potentially unstable regions, we use Tayler's stability criteria (Tayler, 1973) for purely toroidal fields and estimate the stabilisation from the poloidal component, following Braithwaite (2009). In fig. 2 we plot Tayler's criteria for modes $m=0$ and $m=1$ - the $m=0$ mode is unstable almost everywhere and the $m=1$ mode is unstable in a large cone around the poles; however the poloidal field stabilises these modes in most of the meridional plane except near the equatorial plane where it merely stabilises all wavelengths small enough to fit into the available space. We can examine closely the behaviour of the field in the vicinity of the magnetic axis, where it can be 


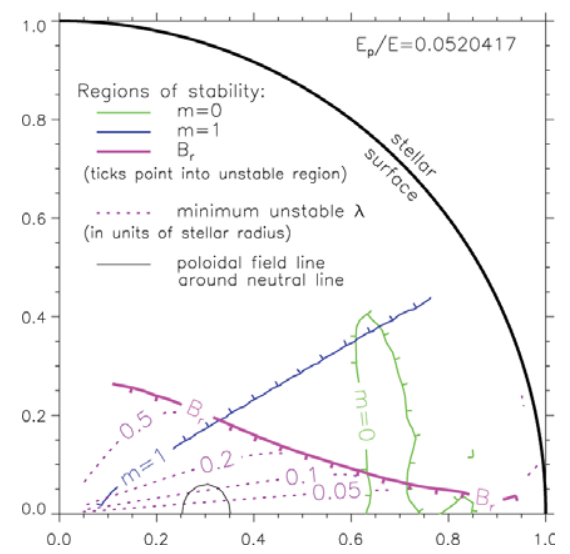

Figure 2. Half of the meridional plane, showing the regions unstable against the $m=0$ and 1 Tayler modes in the absence of the poloidal component.

approximated as the addition of an axial and a toroidal field (cylindrical geometry). Bonanno (2008a) outlined that in this case magnetic configurations can be subject to non-axisymmetric resonant instability. They determined the dependency of the Tayler instability maximum growth rate as a function of the azimuthal wave-number $m$ and of the ratio $\varepsilon$ of the axial field to the toroidal one. In our case, close to the center the flux function exhibits a behaviour in $\Psi \propto r^{2}$, so the azimuthal field is proportional to $s=r \sin \theta$ corresponding to the Bonanno et al.'s parameter $\alpha=1$. As underlined by the authors, in that case the maximum growth rate changes remarkably slowly with $m$ for all modes with $m \geqslant 2$ and the instability is weakly non-anisotropic. If we take as a value for $s_{1}$ the radius of the neutral line or the one where the azimuthal field is strongest, we obtain respectively $\varepsilon=0.64$ or $\varepsilon=0.79$. According to their study (see Bonanno, 2008a; fig. 7), we fulfill the stability criterion for the modes $m=0,1$ and 2 . Our results are therefore in agreement with their linear analysis. In the simulations we run, the mixed configuration has a poloidal energy fraction $E_{\mathrm{p}} / E=0.052$. The magneticto-thermal energy ratio $E / U \approx 1 / 400$, which should mean that for stability we require $E_{\mathrm{p}} / E \gtrsim 0.04$ (Braithwaite, 2009). We see then that this value of $E / U$ is near the upper limit for stability - in other words, we are near the boundary of validity of the weak-field approximation, reinforcing the result.

\section{References}

Bonanno, A. \& Urpin, V. 2008, A\&A, 488, 1

Bonanno, A. \& Urpin, V. 2008, A\& $A, 477,35$

Braithwaite, J. \& Nordlund, ^ 2006, A\&A, 450,1077

Braithwaite, J. \& Spruit, H. 2004, Nature, 431,819

Chandrasekhar, S. 1958, Proc. Nat. Acad. Sci., 44, 842

Duez, V. \& Mathis, S. 2010, A\& A, 517, A58

Duez, V., Braithwaite, J., \& Mathis, S. 2010, ApJL, 524, L34

Montgomery, D. \& Philips, L. 1988, Phys. Rev. A, 38, 2953

Nordlund, A. \& Galsgaard, K. 1995, http://www.astro.ku.dk/ aake/papers/95.ps.gz

Prendergast, K. H. 1956, ApJ, 123, 498

Reisenegger, A. 2009, $A \& \mathcal{E} A, 499,557$

Tayler, R. J. 1973, MNRAS, 161, 365

Taylor, J. B. 1974, Phys. Rev. Lett., 33, 1139

Woltjer, L. 1959, ApJ, 130, 405

Wright, G. A. E. 1973, MNRAS, 162, 339 